\title{
Super Hilltop Inflation
}

\author{
Chia-Min Lin ${ }^{1, *}$ And Kingman Cheung ${ }^{1,2,3, \dagger}$ \\ ${ }^{1}$ Department of Physics, National Tsing Hua University, Hsinchu, Taiwan 300 \\ ${ }^{2}$ Physics Division, National Center for Theoretical Sciences, Hsinchu 300, Taiwan \\ ${ }^{3}$ Division of Quantum Phases \& Devices, School of Physics, Konkuk university, Seoul 143-701, Korea
}

October 29, 2018

\begin{abstract}
In this paper, we consider logarithmic radiative corrections and higher order terms to the supersymmetric hilltop F- and D-term hybrid inflation models. Conventional F- and D-term hybrid inflation only predicts $n_{s} \gtrsim 0.98$. We show that via a positive quadratic and a negative quartic correction the spectral index can be reduced to $n_{s}=0.96$ suggested from latest WMAP result and also cosmic string problem appeared in SUSY hybrid inflation can be solved with mild tuning of the parameters if $\kappa \lesssim 0.01$ for F-term inflation and $g \lesssim 0.05$ for D-term inflation.
\end{abstract}

*cmlin@phys.nthu.edu.tw, ${ }^{\dagger}$ cheung@phys.nthu.edu.tw

\section{Introduction}

Inflation [1, 2, 3] (for review, 4, 5, 6]) is an vacuum dominated epoch in the early universe when the scale factor grew exponentially. This scenario is used to set the initial condition for the hot big bang and provided primordial density perturbation as the seed of structure formation. In the framework of slow-roll inflation, the slow-roll parameters are defined by

$$
\begin{gathered}
\epsilon \equiv \frac{M_{P}^{2}}{2}\left(\frac{V^{\prime}}{V}\right)^{2}, \\
\eta \equiv M_{P}^{2} \frac{V^{\prime \prime}}{V}
\end{gathered}
$$

where $M_{P}=2.4 \times 10^{18} \mathrm{GeV}$ is the reduced Planck mass. The spectral index can be expressed in terms of the slow-roll parameters as

$$
n_{s}=1+2 \eta-6 \epsilon \text {. }
$$

The spectrum is given by

$$
P_{R}=\frac{1}{12 \pi^{2} M_{P}^{6}} \frac{V^{3}}{V^{\prime 2}}
$$


With the slow-roll approximation the value of the inflaton field $\phi$, in order to achieve $N$ e-folds inflation, is

$$
N=M_{P}^{-2} \int_{\phi_{\text {end }}}^{\phi(N)} \frac{V}{V^{\prime}} d \phi .
$$

From observation [7], $P_{R}^{1 / 2} \simeq 5 \times 10^{-5}$ at $N \simeq 60$.

Suppose we are going to build a small field inflation model, i.e. $\phi \underset{\sim}{\sim} M_{P}$. For a wide range of the potentials, for example, polynomial and logarithmic forms, we have

$$
V^{\prime} \simeq \phi V^{\prime \prime}
$$

Therefore, since $\phi \lesssim M_{P}$

$$
|\epsilon|=\frac{\phi^{2}}{2 M_{P}^{2}}|\eta|^{2} \ll|\eta| .
$$

From Eq. (3)

$$
n_{s} \simeq 1+2 \eta
$$

The latest WMAP 5-year result prefers the spectral index around $n_{s}=0.96$ [7] which implies $\eta=-0.02$. This suggests the inflation took place near a maximum of the potential which is concave-downward. This kind of models is called "hilltop inflation" [8, 9].

In hybrid inflation [10], a false vacuum is provided by the waterfall field. By adding the false vacuum, even use a quadratic potential (like the one in chaotic inflation [11]), the model can still be a small field model [12. In the framework of supersymmetry, there are two standard types of hybrid inflation models: F-term [13, 12] and D-term [14, 15, 16, 17]. Both types of models predict the spectral index $n_{s}>0.98$. In conventional forms of models, cosmic strings are produced after inflation. However, there is a $10 \%$ upper bound for the contribution from cosmic strings to the CMB angular power spectrum [18, 19, 20]. This puts a very strong constraint to the parameters in both F- and D-term hybrid inflation. In [21, it was shown that the cosmic string problem for D-term inflation can be solved by using a negative quadratic correction to the scalar potential, but a spectral index $n_{s}<0.96$ is required. In D-term inflation, if we reduce the superpotential coupling to $\lambda \lesssim O\left(10^{-4}-10^{-5}\right)$ and the $U(1)_{F}$ gauge coupling $g \lesssim 2 \times 10^{-2}$ [22, 23], then the cosmic string contribution to the CMB can be reduced to less than $10 \%$. This also makes $n_{s} \simeq 1$. For $10 \%$ cosmic string contribution, $n_{s} \simeq 1$ is actually what we would like to have [24, 25]. However if the cosmic string contribution is further reduced, for example, less than $5 \%, n_{s} \simeq 1$ is not favored. On the other hand, in the F-term inflation, the constraint for the superpotential coupling from cosmic string study is $\kappa \lesssim 7 \times 10^{-7}$ [23, 26]. The above parameters will be defined in the subsequent sections.

It is well-known that there is the $\eta$-problem [12, 27] in the F-term hybrid inflation. But if we tune the coupling mildly, it can be used as ' $\eta$-correction'. If the correction is negative [28], $n_{s}=0.96$ can be achieved. In this paper, we show that we can also have $n_{s}=0.96$ in the case the quadratic correction is positive if we include a negative quartic term. Similar models can be realized in D-term hybrid inflation, where the higher order correction terms can come from non-minimal gauge kinetic function.

It would be interesting if we can have both $n_{s}=0.96$ and solving the cosmic string problem. In this paper, we show that by considering some generic higher order terms, the spectral index can be reduced to fit the data and the cosmic string problem can also be solved.

This paper is organized as follows. In section 2, we introduce a simple parameterization of our model and show the analytic solutions of it. In section 3 , the formation of cosmic string and its 
contribution to the CMB anisotropy is explained. In section 4, we discuss the hilltop version of D-term hybrid inflation. In section 5, we discuss the F-term hybrid inflation case. Finally, we present our conclusions.

\section{The Potential}

In this section, for simplicity, we set the reduced Planck mass $M_{P}=1$. Consider the following form of the scalar potential

$$
V(\phi)=V_{0}\left(1+\frac{a}{2} \phi^{2}-\frac{b}{4} \phi^{4}+c \ln \left(\frac{\phi}{\Lambda}\right)\right)
$$

where $a, b, c>0$. A sketch of the potential is shown in Fig. (11). The case $a=b=0$ corresponds to conventional F- and D-term hybrid inflation. The case $a<0$ has been considered in the framework of F-term [28, 8], D-term [8, 21, 29] and $F_{D}$-term [30] hybrid inflation. The case $c=0$ has been considered in [9, 31].

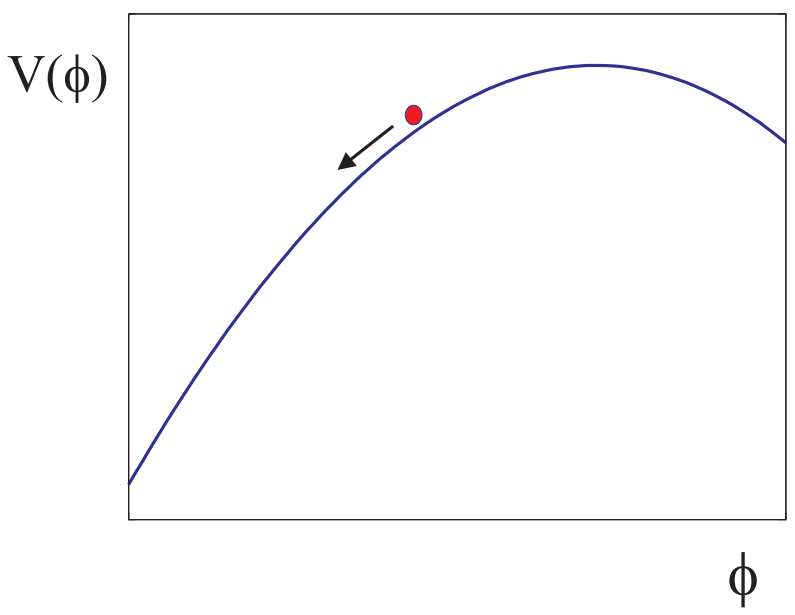

Figure 1: The typical form of $V(\phi)=V_{0}\left(1+\frac{a}{2} \phi^{2}-\frac{b}{4} \phi^{4}+c \ln \left(\frac{\phi}{\Lambda}\right)\right)$ for $a, b, c>0$. The red spot represents the slow-rolling inflaton field.

In order to achieve the slow-roll condition, we require $V \simeq V_{0}$, giving

$$
\begin{gathered}
\frac{V^{\prime}}{V}=a \phi-b \phi^{3}+\frac{c}{\phi} \\
\frac{V^{\prime \prime}}{V}=a-3 b \phi^{2}-c / \phi^{2} \equiv \eta
\end{gathered}
$$

Then Eq. (5) has the analytic solution

$$
\phi^{2}=\frac{(1+B) \sqrt{4 b c+a^{2}}+(B-1) a}{2 b(B-1)}
$$

where

$$
B \equiv A e^{2 \sqrt{4 b c+a^{2}} N}
$$


and

$$
A \equiv \frac{2 b \phi_{e n d}^{2}+\sqrt{4 b c+a^{2}}-a}{2 b \phi_{e n d}^{2}-\sqrt{4 b c+a^{2}}-a} .
$$

The spectral index is

$$
n_{s} \simeq 1+2 \eta
$$

The curvature perturbation is

$$
P_{R}=\frac{1}{12 \pi^{2}} \frac{V_{0}}{\left(a \phi-b \phi^{3}+\frac{c}{\phi}\right)^{2}} .
$$

\section{Cosmic Strings}

In hybrid inflation, when the inflaton rolls down its potential to the critical point, inflation ends via the tachyonic instability of the waterfall field. The potential then goes to its global minimum with the VEV of the waterfall field. After hybrid inflation, there can be a $U(1)$ gauge symmetry broken by the VEV of the waterfall field and thus cosmic strings (for review, see [32]) form via the Kibble mechanism [33. The Kibble mechanism says that when the symmetry breaking happens, different patches (at least smaller than the particle horizon) of the Universe end up with different vacua. At the boundaries between patches, topological defects (in our case, cosmic string) form (Fig. (2) ). The original version of Kibble mechanism describes the symmetry breaking when the Universe cooled down from high temperature. Hybrid inflation is an alternative to achieve the symmetry breaking.

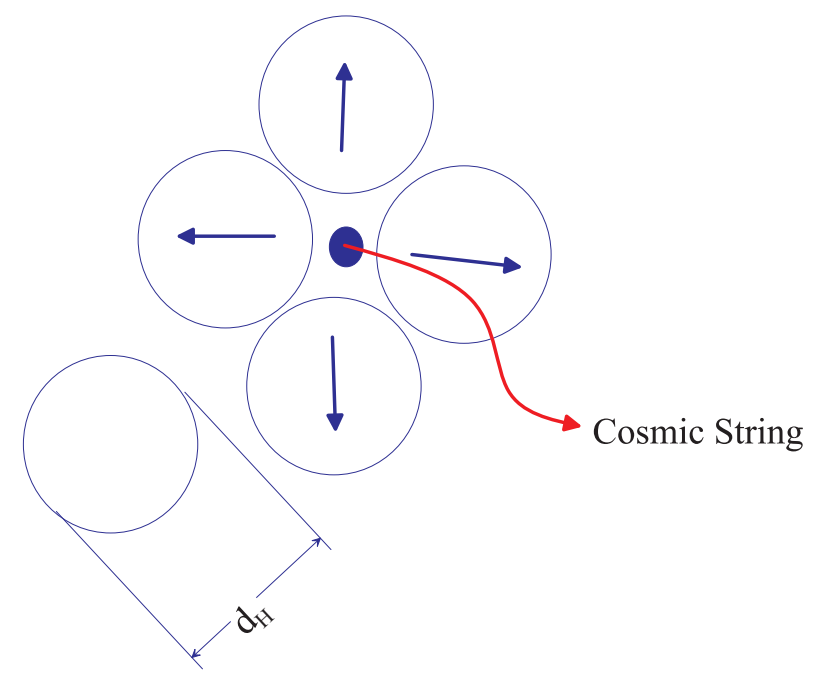

Figure 2: The Universe can be divided into different parts by correlation length no larger than the particle horizon $d_{H}$. Each part has different phase of the field represented by different directions of the arrow, result in cosmic string formation.

The CMB anisotropy is analyzed by decomposing the fluctuation into spherical harmonics given by 34

$$
\frac{\Delta T(\theta, \phi)}{T}=\sum_{l, m} a_{l m} Y_{l m}(\theta, \phi),
$$




$$
C_{l} \equiv \frac{1}{2 l+1} \sum_{m=-l}^{m=+l}\left|a_{l m}\right|^{2},
$$

where $Y_{l m}$ 's are spherical harmonic functions and $C_{l}$ is the angular power spectrum. It is highly nontrivial to calculate the effect of cosmic string network to CMB angular power spectrum, but roughly we can write 22

$$
l(l+1) C_{l}^{s t r .} \propto(G \mu)^{2}
$$

where $G$ is Newton's constant and $\mu$ is the string tension. In [35] the value

$$
G \mu=2.0 \times 10^{-6}
$$

was obtained using a field-theory simulation of the cosmic string network when the cosmic string contribution to CMB angular spectrum is assumed $100 \%$ (at $l=10$ ).

As mentioned in Sec. 1, there is a 10\% upper bound for the contribution of cosmic string network to the CMB angular power spectrum from the observation data. Therefore, the data puts an upper bound on the string tension $\mu$.

\section{D-term Inflation}

The superpotential of D-term hybrid inflation is given by

$$
W=\lambda S \Phi_{+} \Phi_{-}
$$

where $S$ is the inflaton superfield, $\lambda$ is the superpotential coupling, and $\Phi_{ \pm}$are chiral superfields charged under the $U(1)_{F I}$ gauge symmetry responsible for the Fayet-Iliopoulos term. The corresponding scalar potential is

$$
V\left(S, \Phi_{+}, \Phi_{-}\right)=\lambda^{2}\left[|S|^{2}\left(\left|\Phi_{+}\right|^{2}+\left|\Phi_{-}\right|^{2}\right)+\left|\Phi_{+}\right|^{2}\left|\Phi_{-}\right|^{2}\right]+\frac{g^{2}}{2}\left(\left|\Phi_{+}\right|^{2}-\left|\Phi_{-}\right|^{2}+\xi\right)^{2},
$$

where $\xi$ is the Fayet-Iliopoulos term and $g$ is the $U(1)_{F I}$ gauge coupling. A very small $g$ (far smaller than order $O(1)$ ) is regarded as unnatural, because we do not know of any small (for example $g<10^{-3}$ ) gauge couplings in particle physics. The true vacuum of the potential is given by $|S|=\left|\Phi_{+}\right|=0$ and $\left|\Phi_{-}\right|=\sqrt{\xi}$. When $|S|>\left|S_{c}\right|=g \xi^{1 / 2} / \lambda$, there is a local minimum occurred at $\left|\Phi_{+}\right|=\left|\Phi_{-}\right|=0$. Therefore, at tree level the potential is just a constant $V=g^{2} \xi^{2} / 2$. The 1-loop corrections to $V$ can be calculated using the Coleman-Weinberg formula 36]

$$
\Delta V=\frac{1}{64 \pi^{2}} \sum_{i}(-1)^{F} m_{i}^{4} \ln \frac{m_{i}^{2}}{\Lambda^{2}},
$$

with $m_{i}$ being the mass of a given particle, where the sum goes over all particles with $F=0$ for bosons and $F=1$ for fermions and $\Lambda$ is a renormalization scale. Thus the 1-loop potential is given by $(\operatorname{setting} \phi=\sqrt{2} \operatorname{Re}(S))$

$$
V(S)=V_{0}\left(1+\frac{g^{2}}{4 \pi^{2}} \ln \left(\frac{\phi^{2}}{\Lambda}\right)\right)
$$

where $V_{0}=g^{2} \xi^{2} / 2$.

In the framework of supergravity, the D-term scalar potential is given by [4]

$$
V_{D}=\frac{1}{2}(\operatorname{Re} f)^{-1} g^{2}\left(q_{n} K_{n} \Phi^{n}+\xi\right)^{2},
$$


where $K$ is the Kähler potential and $K_{n} \equiv \partial K / \partial \phi$. The gauge kinetic function $f$, which determines the kinetic terms of the gauge and gaugino fields, is a holomorphic function of all the complex scalar fields $\Phi_{n}$. One can choose $f=1$ when the scalar fields are at the origin. Then, for example along the $\Phi_{1}$ direction [37,

$$
1 / f=1+\lambda_{f} \Phi_{1}^{2} / M_{P}^{2}+\cdots,
$$

where the linear term is assumed to be forbidden by a symmetry and $\lambda$ is generically of order $O(1)$. In this paper, we consider

$$
1 / f=1+\alpha \frac{\phi^{2}}{M_{P}^{2}}-\beta \frac{\phi^{4}}{M_{P}^{4}}
$$

where $\phi$ is the inflaton field. Therefore, the scalar potential is

$$
V=V_{0}\left(1+\alpha \frac{\phi^{2}}{M_{P}^{2}}\right)-\frac{V_{0}}{M_{P}^{4}} \beta \phi^{4}+V_{0} \frac{g^{2}}{4 \pi^{2}} \ln \left(\frac{\phi}{\Lambda}\right),
$$

where $g$ is the $U(1)_{F I}$ gauge coupling and $\alpha, \beta$ are generically of order $O(1)$. If $\alpha, \beta$ are far from 1 , fine-tuning is needed.

After inflation the $U(1)_{F I}$ gauge symmetry is spontaneously broken by the VEV of $\Phi_{-}$, cosmic strings form. The mass per unit length of the string is given by

$$
\mu=2 \pi \xi
$$

From Eqs. (20, 29), we have $\xi^{1 / 2}=6.8 \times 10^{15} \mathrm{GeV}$ for the cosmic string contribution to angular spectrum being $100 \%$. We can calculate the upper bound on $\xi$ by demanding the cosmic string contribution to the angular power spectrum to be less than $10 \%$ or $5 \%$. We obtain $\xi^{1 / 2} \leq$ $4 \times 10^{15} \mathrm{GeV}=1.67 \times 10^{-3} M_{P}$ for less than $10 \%$ and $\xi^{1 / 2} \leq 1.34 \times 10^{-3} M_{P}$ for less than $5 \%$ contribution 35 .

The results is shown in Figs. (3) 5 . We plot the contours in $(\alpha, \beta)$ for the spectral index $n_{s}=$ $0.95,0.96,0.97$, and for $\xi^{1 / 2}=0.00167 M_{P}(10 \%$ cosmic string contribution $)$ and $\xi^{1 / 2}=0.00125 M_{P}$ (less than $5 \%$ cosmic string contribution). As we can see in the plots, the spectral index $n_{s}=0.96$ can be achieved by mild tuning between the parameters $\alpha$ and $\beta$. Furthermore, if $g \lesssim 0.05$, cosmic string problem can be solved simultaneously at $\alpha=O\left(10^{-2}\right)$ and $\beta=O(1)$. Whereas $\lambda$ can

be calculated using $\phi_{\text {end }} \equiv \sqrt{2}\left|S_{c}\right|=\sqrt{2} g \xi^{1 / 2} / \lambda$. For example, for $\phi_{\text {end }}=0.1, g=0.05$, and $\xi^{1 / 2}=0.00125$, we obtain $\lambda=8.83 \times 10^{-4}$.

\section{F-term Inflation}

The superpotential of F-term Inflation is

$$
W=\kappa S\left(\Phi_{+} \Phi_{-}-M^{2}\right)
$$

where $S$ is the inflaton field, and $\Phi_{+}, \Phi_{-}$are the symmetry-breaking fields. The scalar potential is then

$$
V=\kappa^{2}\left|\Phi_{+} \Phi_{-}-M^{2}\right|^{2}+\kappa^{2}|S|^{2}\left(\left|\Phi_{+}\right|^{2}+\left|\Phi_{-}\right|^{2} \mid\right)+\frac{g^{2}}{2}\left(\left|\Phi_{+}\right|^{2}-\left|\Phi_{-}\right|^{2}\right) .
$$

Vanishing of the D-terms requires $\left|\Phi_{-}\right|=\left|\Phi_{+}\right|$. From the potential, the true vacuum is given by $|S|=0$ and $\left|\Phi_{+}\right|=\left|\Phi_{-}\right|=M$. When $|S|>\left|S_{c}\right|=M$, a local minimum occurs at $\Phi_{+}=\Phi_{-}=0$, 


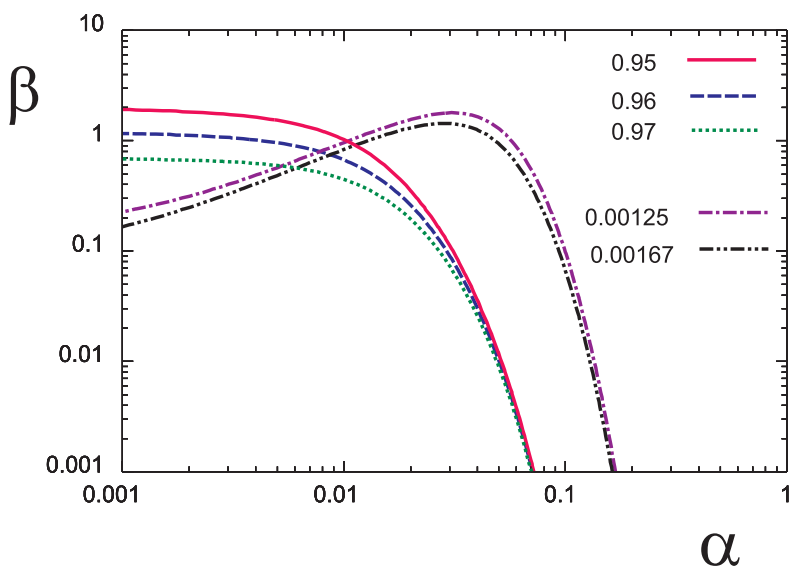

Figure 3: $\phi_{\text {end }}=0.1, g=0.03$

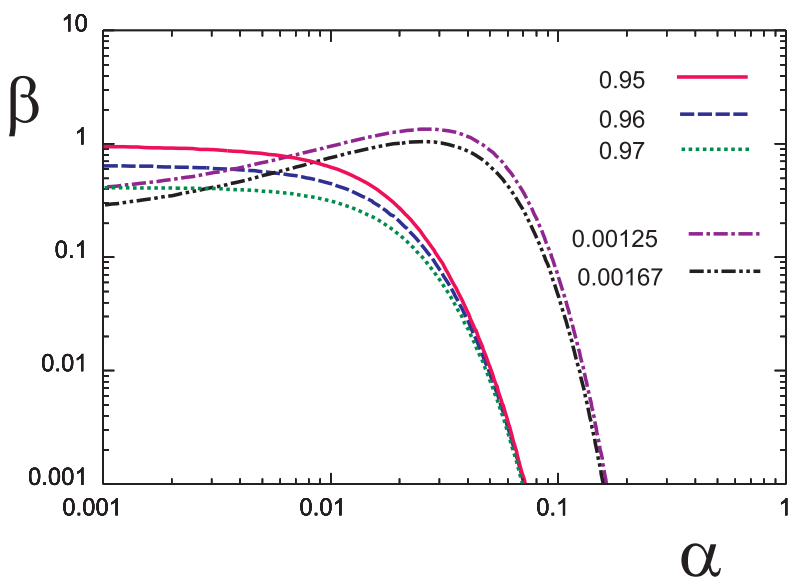

Figure 4: $\phi_{\text {end }}=0.1, g=0.05$

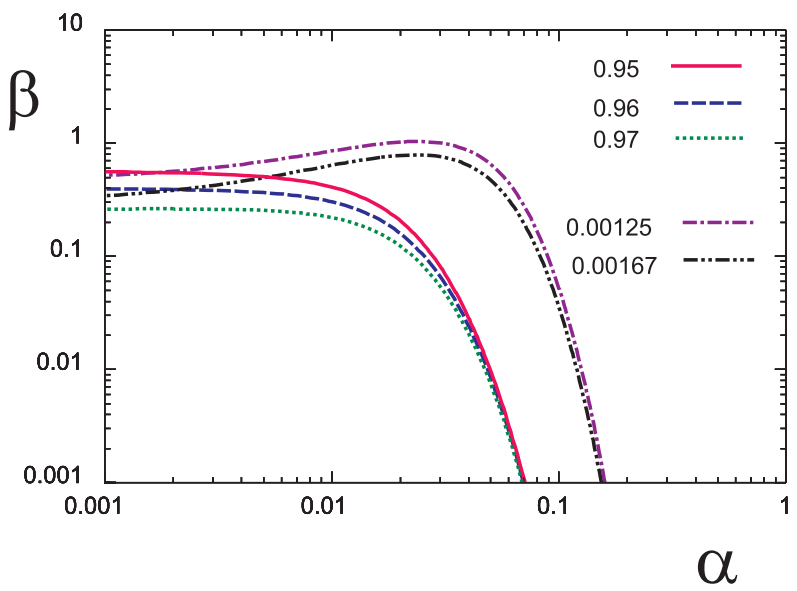

Figure 5: $\phi_{\text {end }}=0.1, g=0.07$ 
and the corresponding potential at tree level is just a constant $V_{0}=\kappa^{2} M^{4}$. The 1-loop-corrected potential is given by $(\operatorname{setting} \phi=\sqrt{2} \operatorname{Re}(S))$

$$
V=V_{0}\left(1+\frac{\kappa^{2}}{8 \pi^{2}} \ln \left(\frac{\phi}{\Lambda}\right)\right)
$$

where $V_{0}=\kappa^{2} M^{4}$ and $\Lambda$ is a renormalization scale. For F-term inflation, $\phi_{\text {end }} \equiv \sqrt{2}\left|S_{c}\right|=\sqrt{2} M$.

Consider a non-minimal Kähler potential [28, 38,

$$
K=|S|^{2}+|\Phi|^{2}+|\bar{\Phi}|^{2}+\kappa_{S} \frac{|S|^{4}}{4 M_{P}^{2}}+\kappa_{S \Phi} \frac{|S|^{2}|\Phi|^{2}}{M_{P}^{2}}+\kappa_{S \bar{\Phi}} \frac{|S|^{2}|\bar{\Phi}|^{2}}{M_{P}^{2}}+\kappa_{S S} \frac{|S|^{6}}{6 M_{P}^{4}}+\cdots .
$$

Here the couplings $\kappa_{S}, \kappa_{S \Phi}, \kappa_{S \bar{\Phi}}$ and $\kappa_{S S}$ are of order $O(1)$, because if they are not, the effective cutoff scale will not be $M_{P}$ but some unknown scales. The SUGRA F-term scalar potential is then given by

$$
V_{F}=e^{\frac{K}{M_{P}^{2}}}\left[\left(W_{m}+\frac{W K_{m}}{M_{P}^{2}}\right)^{\dagger} K^{m^{\dagger} n}\left(W_{n}+\frac{W K_{n}}{M_{P}^{2}}\right)-\frac{3|W|^{2}}{M_{P}^{2}}\right]
$$

where $K^{m^{\dagger} n}$ is the inverse matrix of

$$
K_{m^{\dagger} n}=\partial^{2} K / \partial \phi_{m}^{\dagger} \partial \phi_{n}
$$

During inflation we have

$$
V \simeq \kappa^{2} M^{4}\left(1-\kappa_{S} \frac{\phi^{2}}{2 M_{P}^{2}}+\gamma_{S} \frac{\phi^{4}}{8 M_{P}^{4}}+\frac{\kappa^{2}}{8 \pi^{2}} \ln \left(\frac{\phi}{\Lambda}\right)\right)
$$

where

$$
\gamma_{S}=\left(1-\frac{7 \kappa_{S}}{2}+2 \kappa_{S}^{2}-3 \kappa_{S S}\right)
$$

Equation (36) is of the form in Eq. (9). We define $\alpha \equiv-\kappa_{S} / 2$ and $\beta \equiv-\gamma_{S} / 8$. From $\kappa_{S}$ we can see that $\alpha$ is about order $O(1)$. From $\gamma$ we can see that the natural value of $\beta$ can be as large as something like $O\left(10^{2}\right)$. In [28, 38, a negative quadratic correction is considered (corresponds to negative $\alpha$ ). In this section, we consider positive quadratic correction and negative quartic correction (corresponds to $\alpha, \beta>0$ ).

Similar to D-term inflation, a cosmic string network forms after F-term inflation with the string tension [32, 39]

$$
\mu=2 \pi M^{2} \theta(\beta)
$$

where $\theta(\beta) \sim 2.4 \ln (2 / \beta)^{-1}$ and $\beta \simeq\left(\kappa / g^{\prime}\right)^{2}$, where $g^{\prime}$ is the appropriate gauge coupling, for example, in the framework of GUT, $g^{\prime} \simeq \sqrt{4 \pi / 25}$. The constraint on $M$ is $M \leq 2 \times 10^{15} \mathrm{GeV}=$ $8.3 \times 10^{-4} M_{P}$ under the requirement that cosmic string contributes less than $10 \%$ to the $\mathrm{CMB}$ angular power spectrum [39, 26], while if we require the contribution to be less than $5 \%$ the corresponding constraint becomes $M \leq 7 \times 10^{-4} M_{P}$.

The results are shown in Figs. (6, 6] for various $\kappa$. We plot the contours in $(\alpha, \beta)$ for the spectral index $n_{s}=0.95,0.96,0.97, M=8.3 \times 10^{-4} M_{P}(10 \%$ cosmic string contribution $)$ and $M=6.25 \times 10^{-4} M_{P}$ (less than $5 \%$ cosmic string contribution). As we can see in the plots, $n_{s}=0.96$ can be achieved via mild tuning 1 between the parameters $\alpha$ and $\beta$. Again, if $\kappa<0.01$, cosmic string problem can be solved simultaneously with $\alpha=10^{-1}-10^{-3}$ and $\beta=10-10^{3}$.

\footnotetext{
${ }^{1}$ In the framework of string theory, there is a natural way to obtain small values of $\alpha$ [40, 41].
} 


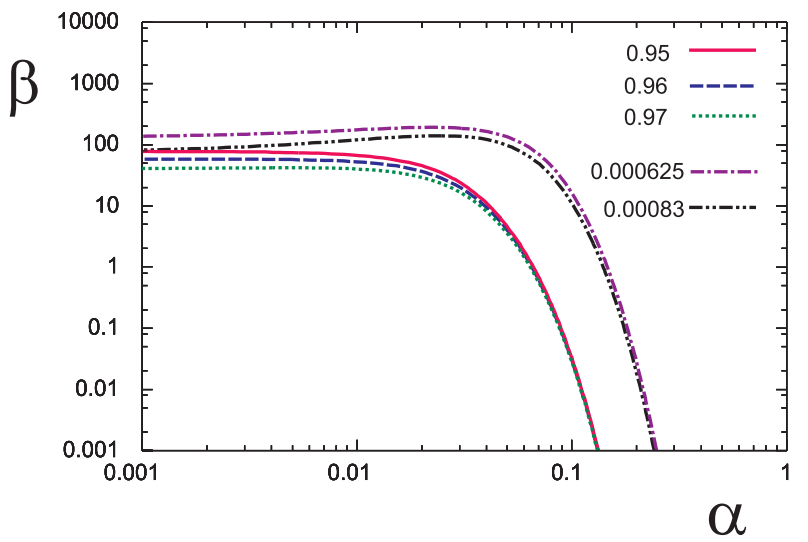

Figure $6: \kappa=0.01$

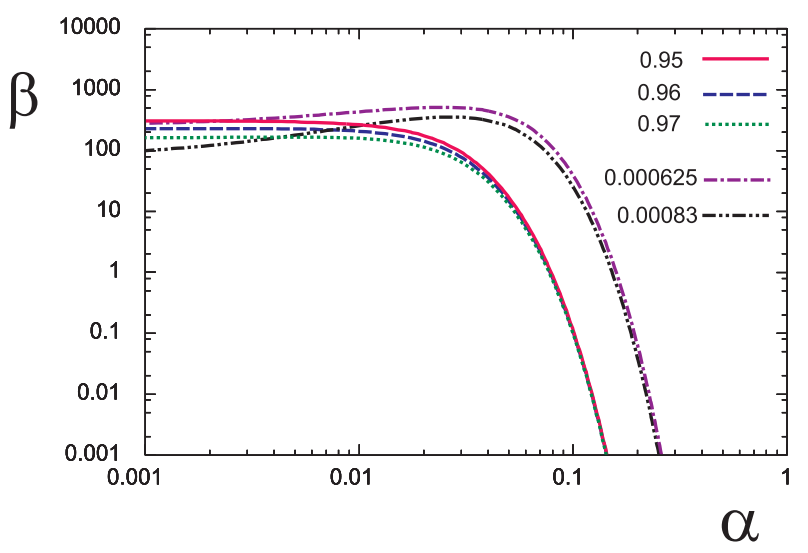

Figure 7: $\kappa=0.005$

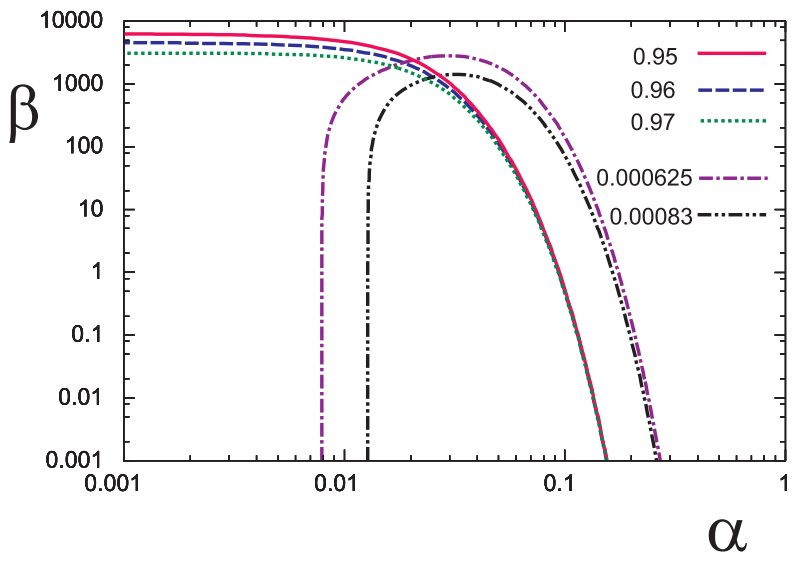

Figure 8: $\kappa=0.001$ 


\section{Conclusions}

In this paper we shown that the higher order corrections to the SUSY hybrid inflation models can have very interesting results if we include quartic terms in addition to the quadratic term. If the quadratic correction is positive and the quartic correction is negative, a hilltop form for the inflaton potential is possible, so that the potential of such a hilltop form makes it possible to have the spectral index reduced to $n_{s}=0.96$ after some mild tuning of the coupling of the higher order terms. For D-term inflation, if $g \lesssim 0.05$, cosmic string problem can be solved with $\alpha=O\left(10^{-2}\right)$ and $\beta=O(1)$. For F-term inflation, if $\kappa<0.01$, cosmic string problem can be solved with $\alpha=10^{-1}-10^{-3}$ and $\beta=10-10^{3}$. Which needs more tuning than the case of D-term inflation.

Lower bounds for $g$ and $\kappa$ are not crucial in this work. The F- and D-term hybrid inflation works even for much lower $g$ or $\kappa$, which means a lower scale $V_{0}$ and smaller $\epsilon$ (a flatter potential). However, this more or less violates the spirit of hybrid inflation which was introduced to prevent small couplings to occur in the successful new inflation [42] or chaotic inflation.

The model proposed in this paper can be applied not only restricted to SUSY hybrid inflation. For example, the D3/D7 model [43] has an effective description as a D-term inflation model and suffers the problem of producing too much cosmic string contribution to the CMB. If higher order terms are generated in this model, cosmic string problem can be evaded.

\section{Acknowledgement}

This work was supported in part by the NSC under grant No. NSC 96-2628-M-007-002-MY3, by the NCTS, and by the Boost Program of NTHU. We are grateful to D. H. Lyth, J. McDonald and Q. Shafi for insightful comments. This work is also supported in parts by the WCU program through the KOSEF funded by the MEST (R31-2008-000-10057-0). 


\section{References}

[1] A. A. Starobinsky, Phys. Lett. B 91, 99 (1980).

[2] K. Sato, Mon. Not. Roy. Astron. Soc. 195, 467 (1981).

[3] A. H. Guth, Phys. Rev. D 23, 347 (1981).

[4] D. H. Lyth and A. Riotto, Phys. Rept. 314, 1 (1999) arXiv:hep-ph/9807278].

[5] D. H. Lyth, Lect. Notes Phys. 738, 81 (2008) arXiv:hep-th/0702128.

[6] A. Linde, Lect. Notes Phys. 738, 1 (2008) [arXiv:0705.0164 [hep-th]].

[7] E. Komatsu et al. [WMAP Collaboration], arXiv:0803.0547 [astro-ph].

[8] L. Boubekeur and D. H. Lyth, JCAP 0507, 010 (2005) arXiv:hep-ph/0502047].

[9] K. Kohri, C. M. Lin and D. H. Lyth, JCAP 0712, 004 (2007) [arXiv:0707.3826 [hep-ph]].

[10] A. D. Linde, Phys. Rev. D 49, 748 (1994) arXiv:astro-ph/9307002.

[11] A. D. Linde, Phys. Lett. B 129, 177 (1983).

[12] E. J. Copeland, A. R. Liddle, D. H. Lyth, E. D. Stewart and D. Wands, Phys. Rev. D 49, 6410 (1994) arXiv:astro-ph/9401011.

[13] G. R. Dvali, Q. Shafi and R. K. Schaefer, Phys. Rev. Lett. 73, 1886 (1994) arXiv:hep-ph/9406319.

[14] P. Binetruy and G. R. Dvali, Phys. Lett. B 388, 241 (1996) arXiv:hep-ph/9606342.

[15] E. Halyo, Phys. Lett. B 387, 43 (1996) arXiv:hep-ph/9606423.

[16] A. Riotto, arXiv:hep-ph/9710329.

[17] D. H. Lyth and A. Riotto, Phys. Lett. B 412, 28 (1997) arXiv:hep-ph/9707273].

[18] L. Pogosian, S. H. H. Tye, I. Wasserman and M. Wyman, Phys. Rev. D 68, 023506 (2003) [Erratum-ibid. D 73, 089904 (2006)] [arXiv:hep-th/0304188].

[19] L. Pogosian, M. C. Wyman and I. Wasserman, JCAP 09, 008 (2004) arXiv:astro-ph/0403268.

[20] M. Wyman, L. Pogosian and I. Wasserman, Phys. Rev. D 72, 023513 (2005) [Erratum-ibid. D 73, 089905 (2006)] arXiv:astro-ph/0503364.

[21] C. M. Lin and J. McDonald, Phys. Rev. D 74, 063510 (2006) arXiv:hep-ph/0604245.

[22] M. Endo, M. Kawasaki and T. Moroi, Phys. Lett. B 569, 73 (2003) arXiv:hep-ph/0304126.

[23] J. Rocher and M. Sakellariadou, Phys. Rev. Lett. 94, 011303 (2005) arXiv:hep-ph/0412143.

[24] N. Bevis, M. Hindmarsh, M. Kunz and J. Urrestilla, Phys. Rev. Lett. 100, 021301 (2008) arXiv:astro-ph/0702223. 
[25] R. A. Battye, B. Garbrecht and A. Moss, JCAP 0609, 007 (2006) arXiv:astro-ph/0607339.

[26] J. Rocher and M. Sakellariadou, JCAP 0503, 004 (2005) arXiv:hep-ph/0406120.

[27] M. Dine, L. Randall and S. D. Thomas, Phys. Rev. Lett. 75, 398 (1995) arXiv:hep-ph/9503303.

[28] M. Bastero-Gil, S. F. King and Q. Shafi, Phys. Lett. B 651, 345 (2007) arXiv:hep-ph/0604198.

[29] C. M. Lin and J. McDonald, Phys. Rev. D 77, 063529 (2008) arXiv:0710.4273 [hep-ph]].

[30] B. Garbrecht, C. Pallis and A. Pilaftsis, JHEP 0612, 038 (2006) arXiv:hep-ph/0605264].

[31] G. German, G. G. Ross and S. Sarkar, Nucl. Phys. B 608, 423 (2001) arXiv:hep-ph/0103243.

[32] M. B. Hindmarsh and T. W. B. Kibble, Rept. Prog. Phys. 58, 477 (1995) arXiv:hep-ph/9411342.

[33] T. W. B. Kibble, J. Phys. A 9, 1387 (1976).

[34] A. R. Liddle and D. H. Lyth,

[35] N. Bevis, M. Hindmarsh, M. Kunz and J. Urrestilla, Phys. Rev. D 75, 065015 (2007) arXiv:astro-ph/0605018].

[36] S. R. Coleman and E. J. Weinberg, Phys. Rev. D 7, 1888 (1973).

[37] D. H. Lyth, Phys. Lett. B 419, 57 (1998) [arXiv:hep-ph/9710347].

[38] M. ur Rehman, V. N. Senoguz and Q. Shafi, Phys. Rev. D 75, 043522 (2007) arXiv:hep-ph/0612023.

[39] R. Jeannerot and M. Postma, JHEP 0505, 071 (2005) arXiv:hep-ph/0503146.

[40] J. A. Casas and G. B. Gelmini, Phys. Lett. B 410, 36 (1997) arXiv:hep-ph/9706439].

[41] J. A. Casas, G. B. Gelmini and A. Riotto, Phys. Lett. B 459, 91 (1999) arXiv:hep-ph/9903492.

[42] A. D. Linde, Phys. Lett. B 108, 389 (1982).

[43] K. Dasgupta, C. Herdeiro, S. Hirano and R. Kallosh, Phys. Rev. D 65, 126002 (2002) arXiv:hep-th/0203019. 